\title{
Infraclavicular brachial plexus stretch injury
}

\author{
Daniel H. Kim, M.D., Judith A. Murovic, M.D., Robert L. Tiel, M.D., \\ AND DAVID G. KLINE, M.D.
}

\author{
Department of Neurosurgery, Stanford University Medical Center, Stanford, California; \\ and Department of Neurosurgery, Louisiana State University Health Sciences Center, \\ New Orleans, Louisiana
}

\begin{abstract}
Object. The authors report the surgery-related results obtained in 143 patients with stretch-induced infraclavicular brachial plexus injuries (BPIs). The entire series comprised 1019 operative BPIs managed at the Louisiana State University Health Sciences Center between 1968 and 1998.

Methods. Infraclavicular lesions represented $143(28 \%)$ of the total of 509 stretch injuries involving both the infraand supraclavicular brachial plexus, of which $366(72 \%)$ were supraclavicular lesions. The operative approach is thoroughly outlined, and common patterns and combinations of involvement of nerves peculiar to the infraclavicular area are presented. Overall, the results of suture and graft repair were favorable for the lateral and posterior cord and their outflows. Repair of medial cord-median nerve also yielded acceptable results. The results of medial cord and medial cord-ulnar nerve, however, were poor. The incidence of associated injuries in the infraclavicular as opposed to the supraclavicular area, including shoulder dislocation and fracture and humeral fractures as well as vascular injuries including axillary artery injury was higher. Results of a literature search supported the finding that vascular injuries were increased due to the juxtaposition of vessels among the brachial plexus elements.

Conclusions. Thus, although less common than their supraclavicular counterpart, infraclavicular stretch injury lesions when they occur are technically more difficult to treat and are associated with a higher incidence of vascular and dislocation/fraction injuries. Favorable results were obtained for lateral and posterior cord lesions and their outflows, with acceptable outcome after medial cord-median nerve stretch injury repair. The results of medial cord and medial cord to ulnar nerve, however, were poor.
\end{abstract}

\section{KEY WORDS • infraclavicular brachial plexus injury $\bullet \quad$ stretch injury $\quad$ nerve graft $\bullet$ neurolysis - anastomosis • nerve action potential}

Injury of the infraclavicular brachial plexus, which comprises cords and their terminal nerves, is most commonly caused by stretch, and this is followed in frequency by GSW and laceration. Iatrogenic insult, another mechanism of injury, results mainly from orthopedic and vascular operations; other less frequent infraclavicular mechanisms include injection, usually of a local anesthetic agent, compression, radiation, and neural sheath tumor.

In this study we focus on the most prevalent mechanism of injury - that of stretch involving the infraclavicular brachial plexus. We report on the surgical treatment of 1019 brachial plexus lesions in patients treated between 1968 and 1998 at LSHUSC. ${ }^{3}$ We also review the literature. Together, these findings form the basis for analysis of this injury mechanism. In this series $509(50 \%)$ of the 1019 lesions were caused by stretch injury, and 143 (28\%) were infraclavicular in location. Thus, although the incidence of infraclavicular injury is lower than that of supraclavicular injury, the number of injuries in this series was sizeable.

Known salient features of stretch-induced infraclavicu-

Abbreviations used in this paper: BPI = brachial plexus injury; LSUHSC = Louisiana State University Health Sciences Center; $\mathrm{NAP}=$ nerve action potential. lar BPI include their nonfocal tendencies and their related involvement of more than one level of the plexus. Additionally there are common patterns and combinations of involvement of nerves that are associated with infraclavicular stretch injuries. Cord and cord-nerve injuries in this region are associated with a higher incidence of other injuries than in those occurring in the supraclavicular region. These associated injuries include shoulder dislocation/fracture and humeral fracture as well as other serious vascular injuries including axillary artery injury. As with the supraclavicular lesions, some of these injuries improve spontaneously, but many do not and thus require surgery.

Infraclavicular surgeries can be technically more demanding than those in the supraclavicular region because there is a need to skeletonize and preserve large vessels such as the axillary and proximal brachial arteries and sometimes veins, which have often been previously repaired and are scarred down.

The purpose of this paper, then, is to look closer at the data obtained from a recent LSUHSC review, specifically focusing on infraclavicular BPIs to determine better if these known features were also demonstrated in the present series. Management guidelines can be established based on the outcomes of this larger case series. 


\section{CLINICAL MATERIAL AND METHODS Patient Population}

Between 1968 and 1998, 1019 patients with BPIs underwent surgery at LSUHSC. Charts were reviewed retrospectively. Patients ranged in age from 4 to 73 years (median age 34 years). Data regarding the injury-related mechanism of stretch to the infraclavicular brachial plexus were analyzed. Electrophysiological studies were also conducted preoperatively. The specific procedure used at LSUHSC and results of intraoperative electrophysiological studies were noted. The minimal follow-up period was 18 months (mean 42 months).

\section{Grading Neural Function}

The LSUHSC grading system is composed of five grades $(0-5$, with Grade 5 indicating the best outcome). Preoperative neural function was graded for each plexal element, and the grades were combined and averaged to yield an overall score for the elements involved. ${ }^{4}$ Successful outcome was represented by Grade 3 or higher. The same method was used to evaluate postoperative neural function. Good and excellent recoveries were represented by Grades 4 and 5, respectively.

\section{Radiological and Electrophysiological Studies}

Most patients underwent preoperative nerve conduction and electromyography studies. Plain radiographs of the shoulder and humerus were important for delineating infraclavicular injuries. In cases in which vascular repair had been previously performed, angiograms were obtained to evaluate arterial patency. Magnetic resonance imaging of the brachial plexus was used to demonstrate the results of trauma to the brachial plexus area. Intraoperatively, direct electrical stimulation was applied above and below the lesion in which there was some degree of physical continuity; NAP recording was also conducted. ${ }^{6}$

\section{Surgical Criteria for Infraclavicular Stretch Injuries}

Patients were usually followed for 3 to 5 months before surgery. Typically this allowed for recovery from the frequently associated vascular and orthopedic injuries and time for adequate soft-tissue coverage if disrupted by the original trauma. In addition, in cases selected for surgery there was no early or significant clinical/electrophysiological reversal of loss. Surgery was more likely to be performed if loss persisted in the distribution of one or more plexal elements that could potentially be helped by operative intervention, especially if repair was necessary. This usually included most of the infraclavicular elements. The major exceptions were cases in which the major deficit was in the medial cord and medial cord-ulnar distributions, where loss was mild or nonexistent in other cord distributions.

\section{Anatomy Involved in Approaches}

The infraclavicular brachial plexus cord and cord-nerve levels are closely involved with the subclavian and axillary arteries and often veins and their branches. This made dissection difficult, especially in the presence of pronounced scarring due to a prior operation and/or severe vascular and osseous injuries.
At LSUHSC the anterior infraclavicular approach to lesions in this area involves an initial curvilinear incision below the clavicle; the incision is continued in or just medial to the deltopectoral groove toward the axilla (Fig. 1). The pectoralis major muscle is split in the direction of its fibers close to the deltoid. Small pectoralis branches and transverse vessels are ligated or cauterized using a bipolar device and divided. The wound is then deepened to the transverse pectoralis minor muscle.

In cases involving proximal cord or focal distal cordnerve procedures, the pectoralis minor muscle can sometimes be partially mobilized and retracted; however, in most cases, it is divided. Deeper dissection in the proximal infraclavicular space usually exposes the lateral cord first, whereas deeper dissection inferior to the lower border of the pectoralis minor muscle typically exposes the median nerve first (Fig. 2). When working from an inferior to a superior location, dissection around the proximal median nerve leads to lateral and medial cord contributions to the median nerve. The site at which the musculocutaneous nerve leaves the lateral cord can vary, and this nerve sometimes even arises from the median nerve. The musculocutaneous nerve is identified by its leash of fine coracobrachialis branches that travel at right angles laterally to the proximal biceps bundle. The musculocutaneous nerve can be dissected down into the biceps brachialis by splitting the overlying muscular fibers in the direction in which they run. This permits mobilization of the nerve usually to the midhumeral level or below.

Shortly after formation of the median nerve from its lateral and medial cord contributions, it is crossed by a transverse vein(s) smaller than the axillary vein. This transverse vein, although sizeable, can be isolated, ligated, and

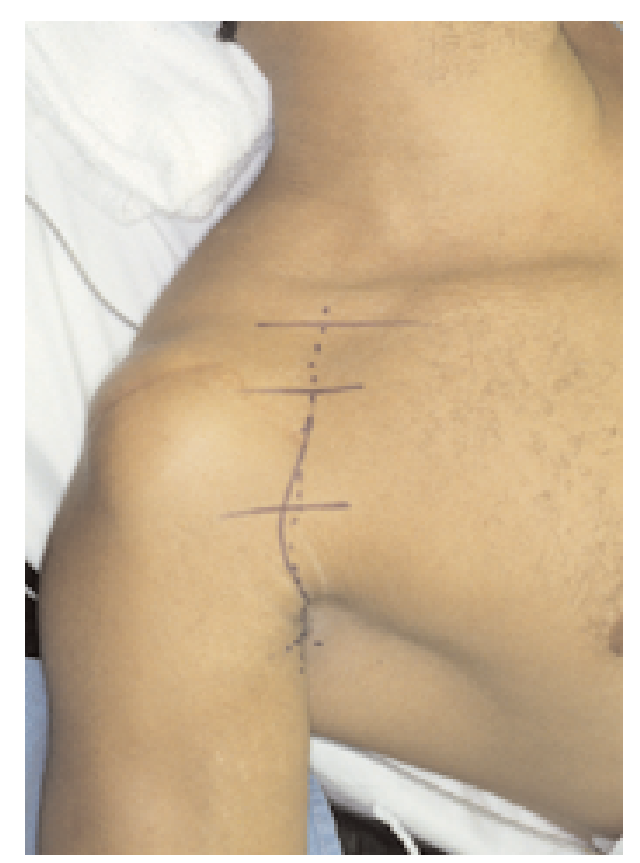

Fig. 1. Photograph showing an example of the incision involved in the approach to the infraclavicular brachial plexus. The incision begins below the clavicle in or just medial to the deltopectoral groove and is extended toward the axilla. 


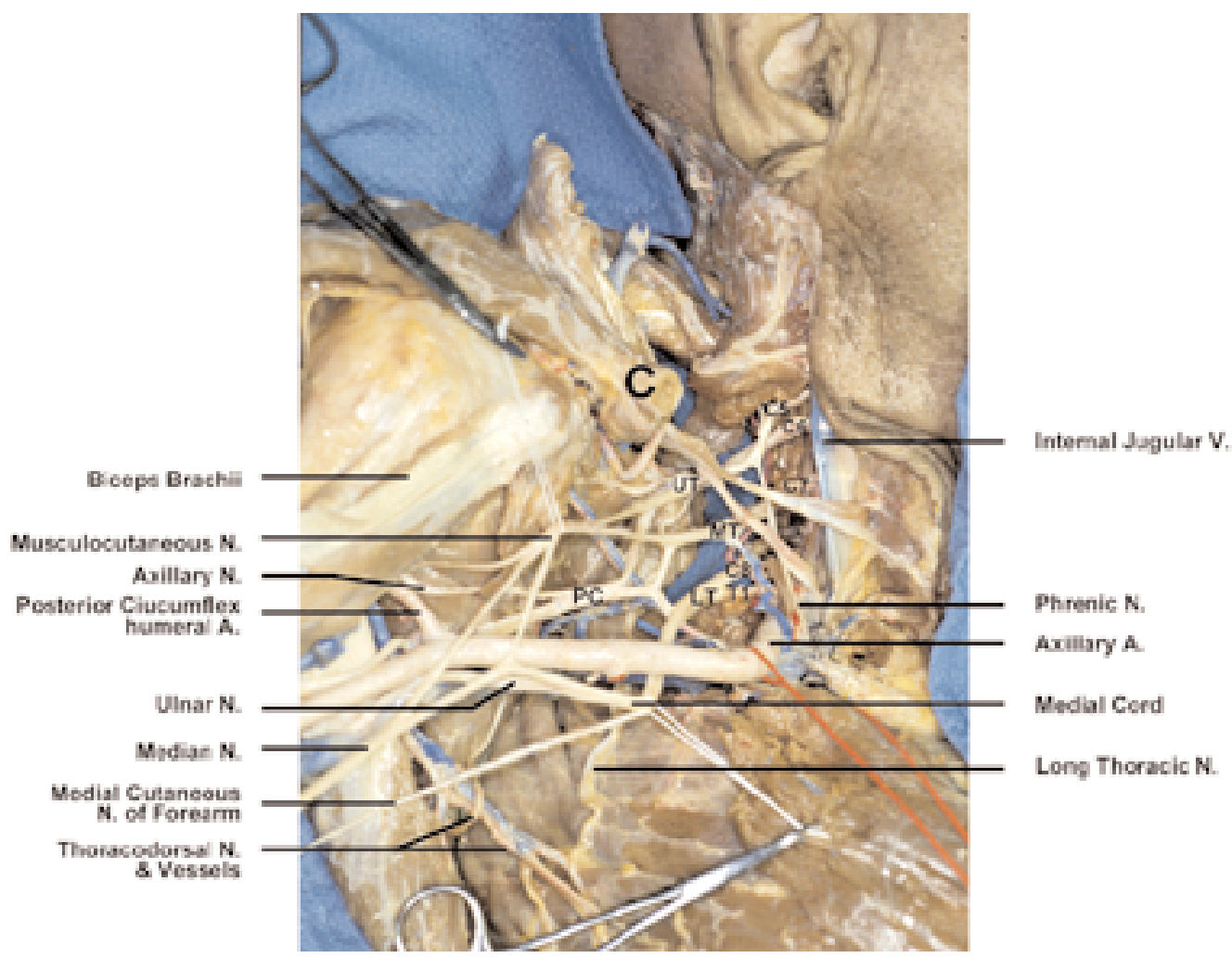

Fig. 2. Photograph of a cadaveric specimen. The right infraclavicular brachial plexus was dissected (medial to lateral view). The clavicle $(\mathrm{C})$ has been removed as has the pectoralis major. $\mathrm{A}=$ artery; $\mathrm{n}=$ nerve; $\mathrm{v}=$ vein.

transected as long as the axillary vein is preserved. Similarly, such a vein can traverse the more proximal lateral cord and require sectioning after ligation. Variations in lateral cord constituency are common and include communications to and from the medial cord before the median and musculocutaneous nerve bifurcations are formed. The lateral cord also usually gives rise to one or more branches to the pectoralis major. Deeper dissection gradually exposes the axillary and brachial arteries. A vein retractor or Penrose drain is used for gentle retraction of the axillary artery to expose the inferolaterally located posterior cord. This element usually gives off the thoracodorsal nerve posteriorly, which runs almost at right angles to the cord to reach the deeper latissimus dorsi muscle.

The posterior cord bifurcates at the same level where the coracobrachialis branch leaves the musculocutaneous nerve, giving rise to the axillary nerve laterally and the radial nerve medially. The latter's relationship to the profundus branch of the axillary artery is key, because here the radial nerve travels medial to this vessel and then tracks to a deeper level to pass superficially to the somewhat fibrous lower border of the subscapularis muscle. In this region, two or three triceps branches may originate to run deeply and somewhat obliquely to the proximal heads of the triceps muscle itself.

Medial cord dissection is helped by full exposure of the axillary artery and other plexal elements at this level, especially the median nerve. If the medial cord contribution to the median nerve is traced proximally, the medial cord itself will be encountered, and this can then be dissected distally to find the origin of the medial pectoral nerve. In addition to these medial pectoral branches, the medial cord gives rise to the antebrachial cutaneous nerves; at the point where the medial cord contribution to the median nerve arises, the ulnar nerve branches. The nerve at this level lies medial and often somewhat posterior to the brachial artery. ${ }^{5}$

\section{RESULTS}

In the LSUHSC series 1019 BPIs were managed operatively between 1968 to 1998 . There were 509 stretch injuries of the brachial plexus of which $366(72 \%)$ were supraclavicular and $143(28 \%)$ were infraclavicular (Table 1 and Fig. 3).

The results confirmed that stretch-related injury was less focal than other mechanisms for injury and less likely to be restricted to one level of the plexal elements alone. Thus, the cords alone seldom sustained the only damage; the damage was more likely to extend from divisions to cords to more distal nerves. If repair was necessary in such instances, outcome was, as expected, not good because of the length and severity of the lesion in continuity.

\section{TABLE 1}

Summary of 509 stretch/contusion-induced BPIs stratified by location

\begin{tabular}{cc}
\hline \hline Injury Location & No. of Lesions (\%) \\
\hline supraclavicular & $366(72)$ \\
infraclavicular & $143(28)$ \\
\hline
\end{tabular}



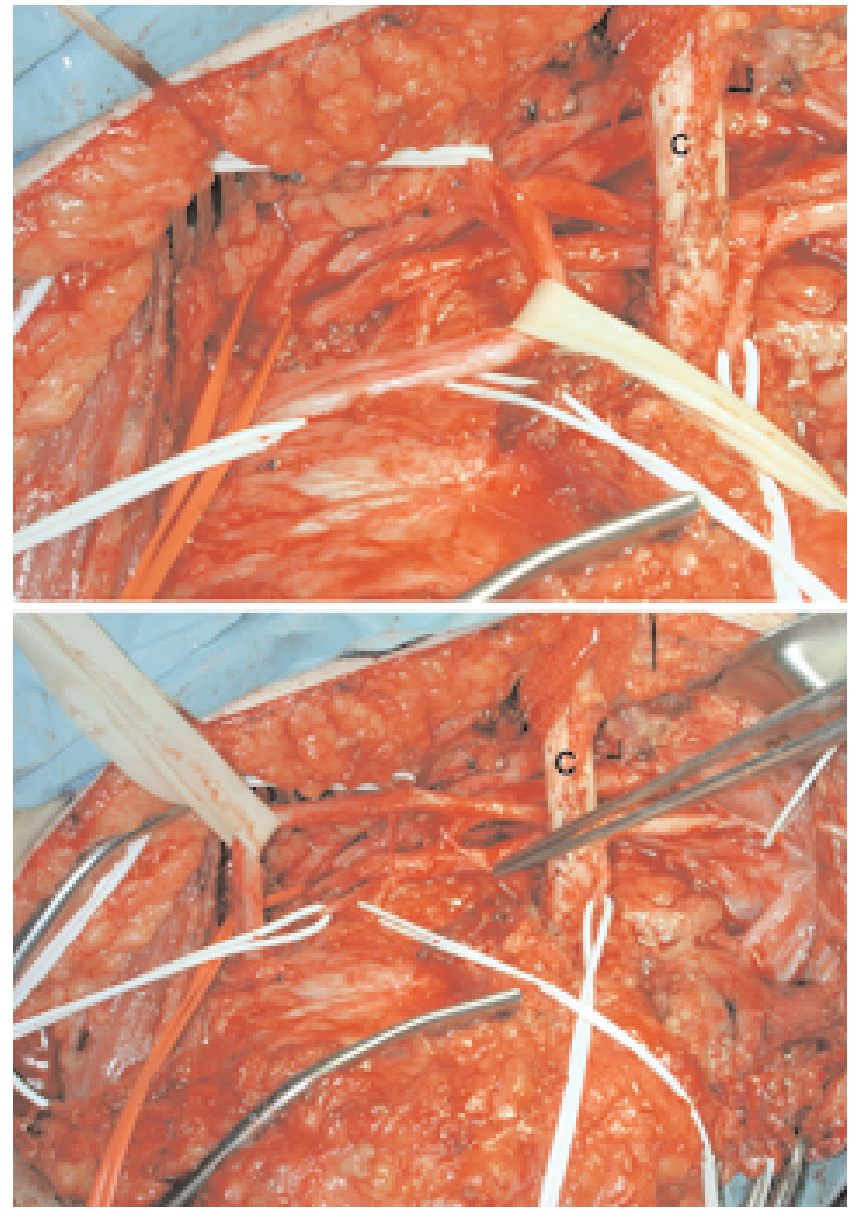

Fig. 3. Intraoperative photographs. Upper: Exploration of the infraclavicular plexus for a stretch injury. Lower: Exploration of the distal branches of the posterior cord, which is pictured at the tip of the forceps. $\mathrm{C}=$ clavical.

\section{Cord Lesions}

If injury was primarily at a division-to-cord or cord level alone, then, for purposes of analysis, these lesions were categorized as those affecting "cords." Of 143 cases of infraclavicular BPIs, 35 operative cases (24\%) involved

\section{TABLE 2}

Summary of surgically treated infraclavicular elements at each level in 143 patients

\begin{tabular}{lc}
\hline \multicolumn{1}{c}{ Injury } & No. of Elements \\
\hline cord lesions & 24 \\
lat & 29 \\
medial & 25 \\
posterior & \\
cord-nerve lesions & 78 \\
posterior-axillary & 65 \\
posterior-radial & 55 \\
lat-musculocutaneous & 49 \\
lat-median & 48 \\
medial-ulnar & 42 \\
medial-median & 415 \\
total & \\
\hline
\end{tabular}

78 elements at the division or cord level (Table 2 and Fig. 4). Dissections of these lesions were difficult because of prior vascular repairs. Repair of lateral cord stretch injuries resulted in mean postoperative functional grades of 4.5 for neurolysis based on the presence of a positive NAP, 4.3 for end-to-end suture repairs, and 3.8 for graft repairs. Medial cord stretch injuries (29 repairs) did not succeed as well. Neurolysis based on the presence of a positive NAP resulted in a mean functional grade of 3.9. End-to-end suture repair was associated with a mean postoperative functional grade of 2.2, and graft-based repairs were less effective (a mean Grade 1.2). Despite difficult dissections due to prior vascular repairs, 25 posterior cord lesions were associated with better results than medial cord lesions. Neurolysis outcomes were represented by a mean grade of 4.1 ; end-to-end suture repair by a mean grade of 3 , and graft repair by a mean grade of 3 .

\section{Cord-to-Nerve Lesions}

If an injury extended beyond the cord level to a proximal peripheral nerve level, it was classified as a "cord-tonerve" lesion. Stretch injuries in the infraclavicular plexus involving cord-nerve were more common than cord lesions. There were 108 patients $(76 \%)$ with 337 infraclavicular cord-nerve lesions that were surgically explored (Table 2). Care was needed to preserve the axillary artery and vein and the profundus brachii artery, especially in cases in which there had been previous dissection with or without vascular repair. It was also important to assess each lesion in continuity by intraoperative NAP recordings. The cumulative mean grades for the lateral cord to its outflows were 4.2 for neurolysis repair, 4 for end-to-end suture repair, and 3 for graft repair. In the patients with 48 injuries involving medial cord-ulnar nerve, a mean grade of 3.6 was achieved if NAPs were detected after neurolysis, even in cases in which initially there was a complete loss of function. Thirteen graft repairs resulted in poor outcomes (mean Grade 1.4). Sixty-five of the elements were posterior cord-radial nerve injuries. Of those, 29 neurolysis procedures resulted in a mean grade of 4.1, and 32 graft repairs yielded a mean grade of 2.7. Of the 78 posterior cord-axillary nerve injuries, 38 were associated with isolated axillary nerve involvement, 11 with suprascapular nerve injuries, 13 with the posterior cord, and 16 with other lesions. Overall, the 28 neurolysis procedures in which an intraoperative NAP was recorded were associated with a mean recovery grade of 4.7 , whereas 48 graft repairs performed in this group yielded a mean grade of 3.5 .

\section{Associated Lesions}

In addition to the associated fractures and vascular lesions already described, isolated axillary palsy or one associated with other cord or cord-nerve injuries was quite common.

\section{Procedural Complications}

An operation often had to be performed despite previous vascular repair. Often, vascular repair had included placement of a graft from the subclavian or axillary artery to the brachial artery (Fig. 4). In these cases especially, 


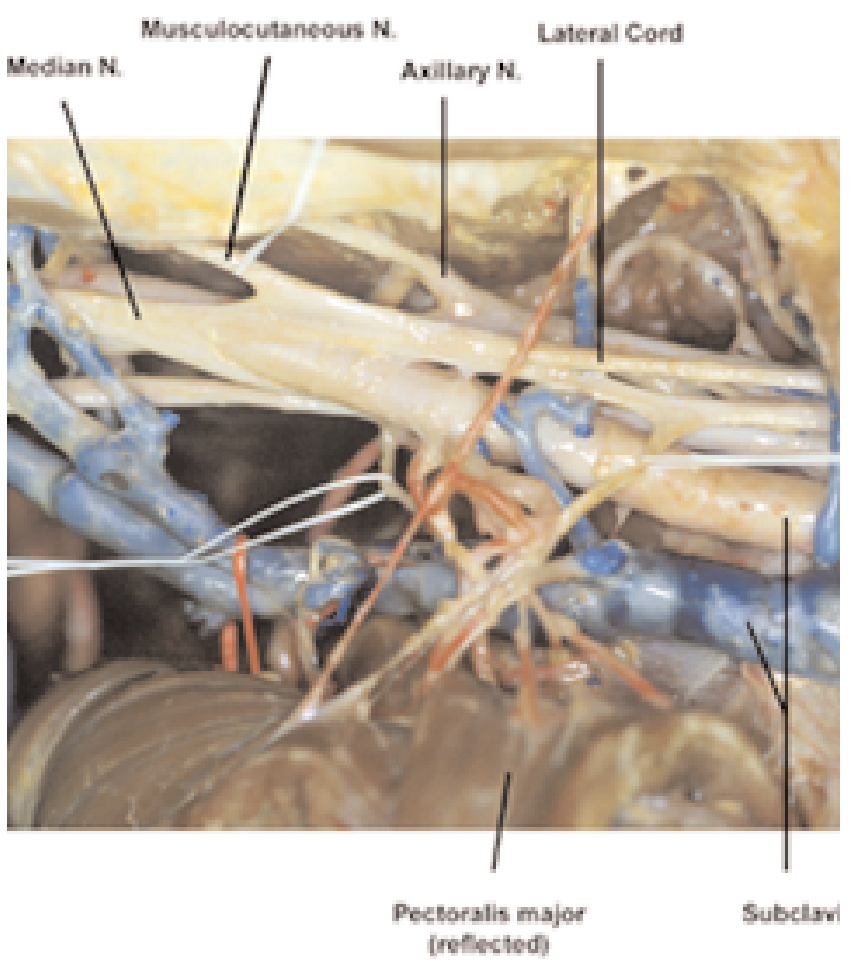

Fig. 4. Photograph of a cadaveric specimen illustrating the proximity of the subclavian vessels to the infraclavicular plexal elements. This results in the higher incidence of associated vascular repairs coinciding with infraclavicular BPIs.

the vessels had to be carefully dissected out and preserved or undergo an additional repair. As a result, these dissections were difficult. Typically, repeated repair was accomplished by the operating service, but sometimes assistance from vascular surgery was necessary. There were also cases in which repeated vascular repair was necessary within 72 hours of the infraclavicular plexus surgery.

Other complications included wound infection, which were superficial and responded to local wound care and antibiotic therapy. A Penrose drain was usually placed at the time of the primary operation, but in one case, a persistent seroma required drainage. Although a few patients suffered increased dysfunction postoperatively, in most of these it was in the lateral cord distribution and it improved spontaneously. In one patient there was an increased radial distribution loss; during follow up triceps, brachioradialis, and wrist extension function improved, but finger and thumb extension remained poor.

\section{DISCUSSION}

Most infraclavicular stretch injuries are caused by motor vehicle accidents, especially those involving motorcycles. ${ }^{7,9}$ Infraclavicular BPIs occurred less frequently than supraclavicular ones, but functional loss can be as severe and often did not reverse with time. This experience varied from earlier observations by other authors. ${ }^{8}$ With regard to prevalence, in the LSUHSC study, the incidence of supraclavicular lesions was $72 \%$ and that of infraclavicular injuries was 28\%. In 1997 Alnot $^{1}$ published a study of 810 patients who underwent surgery between 1975 and 1994; there were 607 cases of supraclavicular lesions
(75\%) and 203 cases of retro- and infraclavicular lesions $(25 \%)$, a ratio that is similar to that in the LSUHSC series evaluated in this paper. There is an important difference, however, because retroclavicular lesions were classified as "cord" lesions in the LSUHSC series. Alnot distinguished two groups of retro- and infraclavicular lesions. There was a group of distal lesions of the terminal branches, which frequently involved the suprascapular, axillary, and musculocutaneous nerves. He also delineated a second group of lesions of secondary trunks, often seen in trauma to the scapular girdle and associated with bone or joint lesions, and sometimes with vascular involvement. Midha9 ${ }^{9}$ reported on 54 patients with BPIs in whom there was also a higher incidence of supraclavicular lesions (29 [62\%]) and lower incidence of infraclavicular lesions (18 [38\%]). In a review by Solonen, et al., ${ }^{12}$ in 52 BPI lesions, $46(88 \%)$ were supraclavicular and six (12\%) were infraclavicular.

In our review, many infraclavicular level stretch injuries did not recover spontaneously and required operative treatment; this observation differs somewhat from those made by previous authors who advocated a nonoperative approach. ${ }^{7,11}$ As with supraclavicular stretch injuries, it helps to sort out the various lesions by using intraoperative NAP monitoring. Lateral and posterior cords and their outflow, such as the musculocutaneous and axillary nerves, can be repaired with surprisingly good results. As expected, medial cord recovery is poor if resection and repair are necessary. Repair of a medial cord-median lesion is, however, useful in the majority of cases.

Stretch-induced infraclavicular BPI tends to be nonfocal and thus can involve more than one level of plexus elements. Therefore, damage was seldom restricted to cords alone and, rather, extended from divisions to cord or from cords to more distal nerves. These findings may be related to the course of the injured individual infraclavicular plexal elements and as well as the fact that these infraclavicular injuries are often caused by associated fractures. They may also be related to sites where the nerve is tethered; for example the musculocutaneous nerve is relatively tethered where it enters coracobrachialis and biceps muscles, whereas the axillary nerve can be tethered as it penetrates the quadrangular space and winds around its point of insertion on the underside of the deltoid muscle.

As noted earlier, there was a high association of infraclavicular brachial lesions with fractures and serious vascular injuries. Some of these injuries improved spontaneously, but many did not and required surgery. In a study of 108 brachial plexus lesions caused by traumatic traction injuries, Narakas ${ }^{10}$ reported on 17 patients with infraclavicular plexal lesions of which seven $(41 \%)$ presented with a rupture of the axillary artery. Birch ${ }^{2}$ described BPIs, in which rupture of the subclavian-axillary artery occurred in only $10 \%$ of supraclavicular and in more than $20 \%$ of infraclavicular lesions. The high association of infraclavicular lesions with fractures and other serious vascular injuries can be attributed to the proximity of this region to the osseous structures of the area and the subclavian and axillary arteries and veins.

\section{CONCLUSIONS}

In this review of infraclavicular lesions of the brachial plexus, we found that these injuries were nonfocal and 
involved more than one level of plexal elements (Table 2). Additionally damage was seldom restricted to cords alone and rather extended from divisions to cords or cords to more distal nerves or from divisions to distal nerves.

A review of the recent literature demonstrated that there is a higher incidence of associated vascular injuries in the infraclavicular injuries than in supraclavicular injuries. This is most likely the result of the juxtaposition of large vessels and the plexus elements.

The results of suture and graft repair were favorable for lateral and posterior cord and their outflows. Medial cordmedian nerve repairs yielded acceptable results, whereas repair of medial cord and medial cord-ulnar nerve resulted in poor outcomes. If resection was not needed in the distribution because NAPs were recorded across a lesion in continuity, then outcome after spontaneous regeneration was surprisingly good.

In summary, lesions in this region exhibit distinctive characteristics such as those related to associated fractures and vascular injuries. The surgical approach must be tailored to the lesion to identify and correct the stretch lesion. Not surprisingly surgical treatment at some levels is more effective than at others with regard to functional outcomes.

\section{References}

1. Alnot JY: Traumatic lesions of the adult brachial plexus. J Bone Joint Surg Br 79-B (Suppl):135-136, 1997

2. Birch R: Brachial plexus injuries. J Bone Joint Surg Br 78: 986-992, 1996
3. Kim DH, Cho YJ, Tiel RL, et al: Outcomes of surgery in 1019 brachial plexus lesions treated at Louisiana State University Health Sciences Center. J Neurosurg 98:1005-1016, 2003

4. Kline DG, Hudson AR: Nerve Injuries: Operative Results for Major Nerve Injuries, Entrapments and Tumors. Philadelphia: WB Saunders, 1995

5. Kline DG, Hudson AR, Kim DH: Atlas of Peripheral Nerve Surgery. Philadelphia: WB Saunders, 2001

6. Kline DG, Happel LT: Penfield Lecture. A quarter century's experience with intraoperative nerve action potential recording. Can J Neurol Sci 20:3-10, 1993

7. Leffert RD: Brachial Plexus Injuries. New York: Churchill Livingstone, 1985

8. Leffert RD, Seddon H: Infraclavicular brachial plexus injuries. J Bone Joint Surg Br 47:9-22, 1965

9. Midha R: Epidemiology of brachial plexus injuries in a multitrauma population. Neurosurgery 40:1182-1189, 1997

10. Narakas AO: Lesions found when operating traction injuries of the brachial plexus. Clin Neurol Neurosurg 95 (Suppl): S56-S64, 1993

11. Seddon HJ: Surgical Disorders of Peripheral Nerves. Edinburgh: Churchill Livingstone, 1972, pp 174-198

12. Solonen KA, Vastamaki M, Strom B: Surgery of the brachial plexus. Acta Orthop Scand 55:436-440, 1984

Manuscript received March 17, 2004.

Accepted in final form April 2, 2004.

Address reprint requests to: Daniel H. Kim, M.D., Department of Neurosurgery, Stanford University Medical Center, Room R-2001, Edwards Building, 300 Pasteur Drive, Stanford, California 943055327. email: neurokim@stanford.edu. 